\title{
COMPARISON BETWEEN LAPAROSCOPIC AND RADIOLOGIC UTERINE ARTERIES OCCLUSION IN THE TREATMENT OF SYMPTOMATIC UTERINE MYOMAS
}

\section{Mohamed Eltotongy, Mohamed El-Negeri, Tarek Shokeir, Mohamed Fawzy, Abdel Megied Mashally}

Department of Obstetrics and Gynecology, Mansoura University Hospitals,

Faculty of Medicine, Mansoura University

\section{ABSTRACT}

Objective: To prospectively compare the effectiveness and safety of the two modalities of uterine arteries occlusion (laparoscopy versus embolization.); as a treatment of symptomatizing uterine fibroids; together with clinical outcome after 6 and 12 months follow up.

Methods: Seventy-six consecutive premenopausal women with symptomatic uterine leiomyomata; non of them desired any further pregnancy. They were randomized to treatment either with laparoscopic occlusion or by radiologic embolization of uterine arteries. The primary outcome was reduction of blood loss from pretreatment to 6 and 12 months postoperatively, as measured by the patients own assessment (subjective). Secondary outcomes included, postoperative pain, complications, secondary interventions, and failures.

Results: Seventy women were included in two groups; 36 and 12-month follow-up data were available for all the patients in each group. The percentage reduction in bleeding assessment did not differe between the treatment groups (88.90\% after laparoscopic uterine arteries occlusion versus $88.51 \%$ after embolization. $P=0.48$ ). Fewer participants in the two groups complained of heavy bleeding after 12 months $(8.57 \%$ compared with $5.7 \%$ with embolization, $\mathrm{P}=.044)$.

Conclusion: Both laparoscopic occlusion and superselective embolization of uterine arteries improved clinical symptoms in the majority of patients. Participants with the radiologic embolization pocedure had more postoperative pain but less heavier menstrual bleeding 12 months afte treatment.

\section{INTRODUCTION}

Uterine leiomyomata, which occur in one of every four to five women during reproductive life; is the most common solid pelvic tumour in women (1) Menstrual abnormalities- especially menorrhagia, which often leads to iron deficiency anacmia - are the most common complications of uterine leiomyomata (1).

Hysterectomy is the most common treatment for symptomatic lciomyomata. Medical treatment combining progestogen and hormonal therapy (gonadotropin releasing hormone agonists) often mects with encouraging but transient results in uterine leiomyomas ${ }^{(2)}$. Surgical procedures including myomectomy and hysterectomy often are needed later during the course of events ${ }^{(3,4)}$. But in addition to the risks associated with surgical procedures, hysterectomy may be a cause of emotional trauma $(5,6)$.

For these reasons, the demand for alternative treatments has increased during the last decade, both by patients and by physicians looking for less invasive procedures. Uterine leiomyoma cmbolization has become one such alternative procedure, and laparoscopic occlusion of uterine vessels is suggested as another alternative.

In a preliminary nonrandomized study, the authors 
reported reduction in menstrual bleeding and reduction of leiomyoma volume after both uterine leiomyoma embolization and laparoscopic occlusion of uterine vessels ${ }^{(7)}$. In the present randomized study, the clinical outcome of these two methods is compared. The reduction in bleeding after 6 and 12 months, as measured by patient' own assessment, was the primary outcome variables. Secondary outcome measures were patients' own assessment of pressure symptom reduction, postoperative pain and nausea registration on visual analog scales, recovery time, complications, secondary interventions, and failures.

\section{MATERIALS \& METHODS}

The study was performed at the Department of Obstetrics and Gynecology and at the Department of Radiology, Mansoura University Hospital. seventy-six premenopausal women were referreds to the university clinic for uterine leiomyomas and menorrhagia or pressure symptoms between January 2005 and December 2007 and were included in this randomized, nonblinded trial. The preliminary treatment results from the randomized patients have been reported previously. Inclusion criteria were the women's own interpretation of increased amounts of bleeding, pressure symptoms, and an expressed desire not to have a hysterectomy. Exclusion criteria were suspicion of malignancy, subserous leiomyomata that could easily be removed by laparoscopic surgery, known adenomyosis, and uterine size exceeding the umbilical level. Submnucous leiomyomata with a diameter of less than $3.5 \mathrm{~cm}$ situated completely intracavitarily or with an intramural extension of more than $50 \%$ were considered more suitable for hysteroscopic resection and were therefore excluded. In addition, women wishing to have children were excluded, as well as those with contraindications for surgery. All eligible patients attended a consultation by a gynecologist, which included a gynecological examination, ultrasonography. The patients were informed about the possible risks and benclits of both treatments. After agrecing to participate, paticnts signed a written informed consent form hefore randomization took place. Randomization of $1: 1$ was undertaken in a total of eight blocks of 10 patients each, using sealed envelopes. Five oenvelopes in each block of 10 were assigned to laparoscopic treatment and five to uterine leiomyoma embolization. The envelopes in each block were closed, mixed, and then numbered. Treatment was decided by drawng the next available envelope in ascending numerical order.

Interventional radiologists performed the embolization procedure. The right femoral artery was punctured and the uterine arteries intubated with a $4 \mathrm{~F}$ Cobra catheter or a microcatheter. In all cases, both arteries were embolized with 355- to 500- micron ployvinyl alcohol particles.

The laparoscopic bilateral occlusion of the uterine arteries was carried out by using the lateral approach to the origin of the uterine artery from the internal iliac artery. In all cases, the uterine artery was closed with either two to three endoclips on each side or bipolar coagulation of the vessels.

The participants were encouraged to use the same type of sanitary pads during the study period. The study participants were surveyed in relation to their present leiomyoma-related symptoms before treatment and after 1 month, 3 months, and 6 months, and 12 month respectively.

A standardized questionnaire was used at all appointments. The amount of bleeding was rated as "little," "moderate," "heavey," or "very heavy." Pressure symptoms, including voiding problems, were recorded as "yes" or "no." Participants were asked to grade changes in amount of blecding and pressure symptoms as better, worse, or unchanged. 
total relief of symptoms at the 12-month follow-up was defined as little or moderate bleeding and no pressure symptoms. Clinical failure was defined as persisting symptoms requiring secondary treatment or no improvement at the 6-month follow-up visit. Postoperative pain and nausea during the hospital stay were recorded on post operative chart. The resident doctors were asked to fill in the level of pain and nausea they experienced with patients every 4 hours during the first 24 hours in the hospital and every 6 hours during the next 24 hours or until the patient left the hospital. The analgesic regime consisted of non-steroidal anti-inflammatory drugs and a paracetamol- codeine combination in fixed doses.

Adverse events were also recorded for each patient during the hospital stay and during outpatient visits afer 1 month, 3 months, 6 and 12 months. All subsequent surgical and medical interventions, as well as readmission to the hospital or prolonged hospitalization, were recorded as adverse events.

Statistical analyses were performed with SPSS 12.0 and the data are presented as mean values for normally distributed data and as median values for skewed data. A two-sided $t$ test was used for comparisons of a continuous variable in two patient groups if the variable in question did not have a markedly skewed distribution. If the distribution was markedly skewed, a two-sided Wilcoxon-MannWhitney test was used. A significance level of .05 was used for all tests.

\section{RESULTS}

Of the 76 patients randomized and included in the study, 70 patients received treatment, 35 with uterine arterics embolization and 35 with bilateral laparoscopec occlusion of uterine vessels. All participants in each group completed the 12-month trial. The two groups were similar with respect to age, body mass index, parity, and baseline symptoms (Table I).

Clinical failure was seen in $4(11.43 \%)$ subjects after uterine leiomyoma embolization and in three $(8.57 \%)$ subjects after laparoscopic occlusion. There was no statistical difference between the two groups $(P=0.13)$. However, there were five women still reporting heavy or very heavy bleeding 6 months after treatment, two $(6.45 \%)$ after uterine leiomyoma embolization and three $(9.38 \%)$ after Iaparoscopic treatment. The difference in the intention-to-treat analysis with regard to the number of patients who reported heavy bleeding was statistically significant $(P=.044)$. Three of these patients, one in the uterine leiomyoma embolization group and two in the laparoscopy group, did not meet the definition of clinical failure. All three participants reported imprvement of symptoms, together with reductions in their bleeding and received no additional treatment during the study period. If these three participants had been added to the group of patients deemed clinical failures, there would have been five (14.29\%) patients after uterine leiomyoma embolization and five $(14.29 \%)$ after laparoscopy with unfavorable results 12 months after treatment $(P=.094)$.

Significantly more pain nd nausea were observed after uterine leiomyoma embolization than after laparoscopy (Figure 1). The median amount of analgesics used after the embolization procedure was four times higher than after laparoscopic surgery: 46 mg compared with $12 \mathrm{mg}(P<.001)$.

There was no significant difference in blecding reduction between the treatment groups one month after treatment $(P=0.96)$. At the 3-month follow-up, the subjects treated by uterine arteries embolization had a mean of blecding assessment reduction of $88.57 \%$ as opposed to $88.90 \%$ for those treated by laparoscopy (Table IIA \& B). Six months after utcrine lciomyoma cmbolization, the reduction was 
$87.6 \%$, and the corresponding reduction for laparoscopic treatment was $85.7 \%$ (Table IIa, IIb).

The number of patients reporting reduced menstrual bleeding, reduction of pressure symptoms, or total relief of all symptoms after treatment did not differ significantly between the two treatment groups (Table IV).

Only minor in-hospital adverse events were ohserved. The patients were scheduled to stay for up to 48 hours in the department after treatment.

Twenty-two patients noticed increased vagmal discharge during the postoperative persod. No significant differences were observed in the proportion of women with vaginal discharge extending to 7 days, The most serious adverse events, unilateral deep vein thrombosis, occurred in one patient who was readmitted to the hospital 1 week after laparoscopic bilateral occlusion.

Table (I): Baseline Parameters of the studied groups.

\begin{tabular}{|l|c|c|}
\hline & LOUA (No 35) & UAE (No 35) \\
\hline Age (Years) & $38.4 \pm 5.19$ & $37.38 \pm 5.2$ \\
\hline BMI & 24 & 23.5 \\
\hline parity & $3 \pm 1.9$ & $3 \pm 1.2$ \\
\hline Preoperative hemoglobin gm/100 & $10.5 \pm 0.5$ & $11 \pm 0.3$ \\
\hline No. of patients with menorrhagia & $25(71.45)$ & $20(57.14)$ \\
\hline No. of patients with metrorrhagia & $10(28.59 \%)$ & $15(42.86 \%)$ \\
\hline No of patients with pressure symptoms & 25 & 30 \\
\hline $\begin{array}{l}\text { No of patients with menorrhagia \& pressure } \\
\text { symptoms }\end{array}$ & 30 & 32 \\
\hline
\end{tabular}

LOUA = laparoscopic occlusions of the uterine arteries

$\mathrm{UAE}=$ utcrine artery embolizatıon

Table (IIA): Improvement of LOUA Bleeding Pattern after one month.

\begin{tabular}{|l|c|c|c|c|c|c|}
\hline \multirow{2}{*}{ Improved } & \multicolumn{2}{|c|}{ Menorrhagia } & \multicolumn{2}{c|}{ Metrorrhagia } & \multicolumn{2}{c|}{ Total } \\
\cline { 2 - 7 } & No & $\%$ & No & $\%$ & No & $\%$ \\
\hline Slight & 6 & 24 & 2 & 20 & 8 & 22.2 \\
\hline Significant & 12 & 48 & 2 & 20 & 14 & 38.9 \\
\hline Complete & 5 & 20 & 5 & 50 & 10 & 27.8 \\
\hline Total & 23 & 92 & 9 & 90 & 32 & 88.9 \\
\hline Not improved & 2 & 8 & 1 & 10 & 3 & 8.57 \\
\hline
\end{tabular}

$P$ value 0.094

LOUA : Laparoscopic occlusion of uterne artertes 
Table (IIB): Symptomatic Improvement in Embolization group after one month.

\begin{tabular}{|l|c|c|c|c|c|c|}
\hline \multirow{2}{*}{ Improved } & \multicolumn{2}{|c|}{ Menorrhagia } & \multicolumn{2}{c|}{ Metrorrhagia } & \multicolumn{2}{c|}{ Total } \\
\cline { 2 - 7 } & No & $\%$ & No & $\%$ & No & $\%$ \\
\hline Slight & 4 & 20 & 2 & 13.33 & 6 & 17.14 \\
\hline Significant & 9 & 45 & 7 & 46.66 & 16 & 45.71 \\
\hline Complete & 6 & 30 & 3 & 20 & 9 & 25.71 \\
\hline Total & 19 & 95 & 12 & 90 & 31 & 88.57 \\
\hline Not improved & 1 & 5 & 3 & 10 & 4 & 11.43 \\
\hline
\end{tabular}

$P$ value 0.480

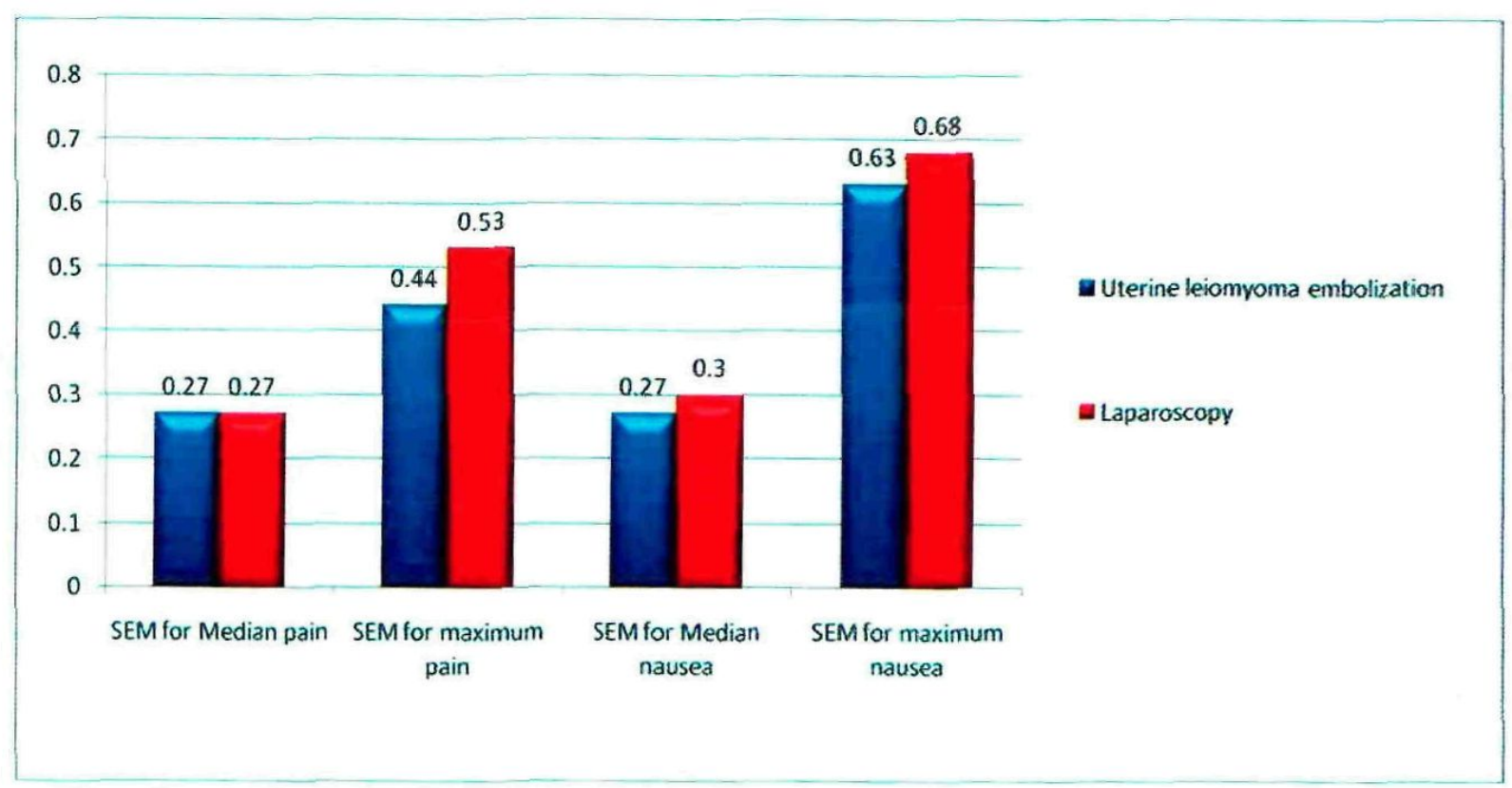

Fig. 1.: Pain and nausea recorded in the first 48 hours after treatment.

$\mathrm{SEM}=$ standard error of the mean

Table (III): Adverse events after treatment one, 3,6 and 12 months follow up.

\begin{tabular}{|l|c|c|c|c|c|c|c|}
\hline & \multicolumn{3}{|c|}{ LUAO } & \multicolumn{3}{c|}{ UAE } & P \\
\cline { 2 - 8 } & $3 \mathbf{m}$ & $\mathbf{6 m}$ & $\mathbf{1 2 m}$ & $\mathbf{3 m}$ & $\mathbf{6 m}$ & $\mathbf{1 2 m}$ & 1.00 \\
\hline Menorrhagia & 0 & 2 & 2 & 0 & 1 & 1 & 1.00 \\
\hline Metrorrhagia & 0 & 1 & 1 & 0 & 1 & 1 & 0.19 \\
\hline Recurrent pressure symptoms & 0 & 2 & 2 & 1 & 2 & 2 & 0.12 \\
\hline Total & 0 & 5 & 5 & 1 & 4 & 5 & 0 \\
\hline
\end{tabular}


Table (IV): Clinical outcome after treatment after one year follow up

\begin{tabular}{|l|c|c|c|}
\hline & UAE $(\mathbf{n = 3 5})$ & LOUA $(\mathbf{n = 3 5})$ & 1 \\
\hline Bleeding reduction & 32 & 31 & 0.69 \\
Reduction of pressure symptoms & 26 & 23 & 0.88 \\
Total relief of pressure & 22 & 15 & 0.15 \\
All symptoms completely resolved & 26 & 21 & 0.18 \\
Satisfaction rate & 33 & 30 & 0.23 \\
Clinical failure & 4 & 3 & 0.13 \\
\hline
\end{tabular}

LOUA = laparoscopic occlusions of the uterine arteries

$\mathrm{UAE}=$ uterine artery embolization

\section{DISCUSSION}

The primary goal of the' present study was to compare the improvement in bleeding patterns between uterine leiomyoma embolization and laparoscopic occlusion. No significant difference in symptom reduction was found between the two treatment options 12 months after treatment.

In the literature, there is one additional study using Pictorial Bleeding Assessment Chart reduction in 76 patients treated by embolization and reported improvement in $55.6 \%$ after 3 months and $58.1 \%$ after 6 months, which is significantly less than in this study ${ }^{(8)}$.

The patients' own assessment of symptom relief is more commonly used as an outcome parameter than the Pictorial. Bleeding Assessment Chart, even though there is a lack of uniformity in defining degree of improvement. Notwithstanding these limitations, the present findings, based on the patients own assessment 6 months after uterine leiomyoma artery embolization, are similar to those of other studics $^{(2,3,5,6,8)}$. The reduction of menstrual bleeding symptoms in $88.57 \%$ and $88.9 \%$ of the patients, after uterine leiomyoma embolization and laparoscopic occlusion respectively in this study is in accordance with the short-term results of the two largest prospective signle-center studies evaluating uterine leiomyoma embolization to date, which reported improvements in menorrhagia in $89 \%$ of patients after 6 months and in $84 \%$ after 16 months, respectively ${ }^{(6,8)}$. Furthermore, the reduction in the amount of bleeding in $88.9 \%$ of the patients after bilateral laparoscopic occlusion in this study is similar to that of other studies, which report improvement of these symptoms in about $90 \%$ of the participants.

In contrast to the lack of difference between the two treament groups with regard to the percentage reduction of patients own assessement of bleeding, significantly more participants reported heavy or very heavy bleedng 6 months after laparoscopic treatment. One possible explanation for this discrepancy might be differences in assessment of bledding.

There are several possible explanations for the slightly less favorable results after the laparoscopic treatment. In the literatures; angiographic and surgical studies have shown numerous anatomical variations of the uterine arteries ${ }^{(9,10)}$. It is thus possible to occlude the wrong artery in laparoscopy or to overlook one artery in cases where there are two instead of one single artery on one sidc. The reason for the failure was found to be insufficient occlusion of the utcrine artery at one side.

The collateral arterial supply to the uterus in general could explain smaller amounts of pain after 
surgical uterine artery occlusion, in spite of ischemia and infarction of leiomyomata. Because embolization is a more distal occlusion reducing collateral flow, increased uterine ischemia can be expected, resulting in increased pain and potentially increased efficacy compared with proximal surgical occlusion. Cncerns have been expressed among interventional radiologists that leiomyomata that are not completely infarcted will have the potential to regrow ${ }^{(11)}$. In two prospective studies, $16 \%{ }^{(12)}$ and $20 \%{ }^{(13)}$ recurrence was found 5-7 years after embolization. If smaller amounts of pain after laparoscopy were caused by less icschemia and thus incomplete infarction of leiomyomata, one would expect even more recurrences with time after laparoscopic occlusion.

The proportion of patients requiring secondary surgery was 4 (11.34\%) after UAE and 3 (8.57) in LOUA groups in our study. This proportion is in accordance with other reporting outcomes after uterine leiomyoma embolization or laparoscopic occlusion. In two larger studies, subsequent interventions or readmission occurred in $10.5 \%$ after 21 months $^{(6)}$ and in $7.5 \%$ after 17 months, ${ }^{(8)}$ respectively. In both of these studies, the main indications for secondary surgery were similar to those in this study: continuous menorrhagia or symptoms related to leiomyoma expulsion. Patient selection is probably most significant for beneficial results, and more careful selection might reduce the number of cases of secondary surgery caused by persistent menorrhagia or expulsion of leiomyomata.

Based on this study, both laparoscopic occlusion of the uterine vessels and embolization appear to improve symptoms associated with uterine leiomyomata in the majority of patients. The laparoscopic procedure resulted in less postoperative pain and nausea and shorter hospital stays, although significantly more participants experienced heavy menstrual bleeding 6 months after laparoscopic occlusion, indicating a more favorable effect after uterine leiomyoma embolization. In the light of these results and bearing in mind the significant risk of surgical complications and lack of long-term results, considering the fact that the laparoscopic techniques are in the field of gynecologic srugery, laparoscopic uterine artery occlusion is likely to attract considerable interest as an effective alternative to hysterectomy treatment of symptomatic uterine leiomyomata.

In order to perform a successful laparoscopic uterine artery occlusion; the surgeons has to be equipped with the necessary laparoscopic skills and should be able to precisely locate the uterine artery in order to correctly ligate the vessels. If these prerequisites are not met massive bleeding may ensue necessitating laparotomy; so laparoscopic procedure for leiomyoma treatment should be confined to centers with appropriate expertise in laparoscopic surgery.

\section{REFERENCES}

1. Vollenhoven $\mathrm{Bj}$ Lawrence AS. Healy D. Uterine fibroids: a clinical review. $\mathrm{Br} \mathrm{J}$ Obstet Gynecol 1990; 97: 285-298.

2. Worthington-Kirsch RL, Popky GL, Hutchins FL. Uterine arterial embolization for the management of leiomyomas: quality-of-life assessment and clinical response. Radiology 1998; 208: 225-229.

3. Goodwin SC, McLucas B, Lee M, Chen G, Perrella $R$, Vedantham $S$, et al. Uterine artery embolization for the treatment of uterine leiomyomata midterm results. J Vasc Interv Radiol 1999; 10: 1159-1165.

4. Healy $\mathrm{D}$, Vollenhowven $\mathrm{Bj}$. The role of $\mathrm{GnRH}$ agonists in the treatment of uterine fibroids. $\mathrm{Br} \mathrm{J}$ Obstet Gynecol 1992; 99: 23-26.

5. Pelage JP, Le Dref O, Soyer P, Kardache M, Dahan H, Abitbol M, et al. Fibroid-related menorrhagia: treatment with superselective embolization of the uterine arteries and midterm follow-up. Radiology 200; 215: 228-231. 
6. Spies JB, asche SA, Roth AR, Kim J, Levy EB, Gomez-Jorge J. Uterine artery embolization for leiomyomata. Obstet Gynecol 2001; 98: 29-34.

7. Hald K, Langebrekke A, Klow NE, Noreng HJ, Bere $A B$, Istere $O$. Laparoscopic occlusion of uierine vessels for he treatment of symptomatic fibroids: initial experience and comparison to uterine artery embolization. Am J Obstet Gynecol 2004; 190: 37-43.

8. Spis JB, Cooper JM, Worthington-Kirsch R, Lipman JC, Mills BB, Benenati JF. Outcome of uterine embolization and hysterectomy for leiomyomas: results of a multicenter study. Am J Obstet Gynecol 2004; 191: 22-31.e

9. Spi S JB, Cooper JM, Worthington-Kirsch R, Lipman JC, Mills BB, Benenatic JF. Outcome of uterine embolization and hysterectomy for leiomyomas: results of a multicenter study. Am J Obstet Gynecol 2004; 191: 22-31.e

10. Walker WJ, Pelage JP. Uterine artery embolisation for symptomatic fibroids: clinical results in 400 women with imaging follow up. BJOG $20(12 ; 109$ : 1262-1272.

11. Holub Z, Lukac J, Kliment L, Urhanck S. Variability of the origin of the uterine artery: laparoscopic surgical observation J Obstct Gynaccol Res 2005; 31: 158-163.

12. Gomez-Jorge J, Keyoung A, Levy EB, Spies JB. Uterine artery anatomy relevant to uterine leiomyomata embolization. Cardiovase Intervent Radiol 2003; 26: 522-527.

13. Holub Z, Lukac J, Kliment L, Urbanek S. Variability of the origin of the uterinee artery: laparoscopic surgical observation. J Obstet Gynaecol Res 2005; 31: 158-163.

14. Iverson RE Cheimow D. Strohbehn K, Waldman L, Evantash EG. relative morbidity and mortality of abdominal hysterectomy and myomectomy for management of uterine leiomyomas. Obstet Gynecol 1996; 88: 415-419. 